\title{
Transmuted Generalized Linear Exponential Distribution
}

\author{
I. Elbatal \\ Institute of Statistical Studies and Research \\ Department of Mathematical Statistics \\ Cairo University.
}

\author{
L.S. Diab \\ College of Science for (girls) \\ Dept. of Mathematics, \\ Al-Azhar University, Egypt.
}

\author{
N. A. Abdul Alim \\ College of Science \\ Dept. of Mathematics \\ Al-Azhar University, Egypt.
}

\begin{abstract}
The linear exponential distribution is a very well-known distribution for modeling lifetime data in reliability and medical studies. We introduce in this paper a new four-parameter generalized version of the transmuted generalized linear exponential distribution. We provide a comprehensive account of the mathematical properties of the new distributions. In particular, A closed-form expressions for the density, cumulative distribution ,quantile and median of the distribution is given. Also, the $r_{t h}$ order moment and moment generating function are derived. The maximum likelihood estimation of the unknown parameters is discussed. Real data are used to determine whether the TGLED is better than other well-known distributions in modeling lifetime data or not.
\end{abstract}

\section{Keywords:}

Transmuted generalized linear exponential distribution, quantile and median, Maximum likelihood estimation, Moments.

\section{INTRODUCTION AND MOTIVATION}

The linear exponential distribution is also known as the Linear Failure Rate distribution, having exponential and Rayleigh distributions as special cases, is a very well-known distribution for modeling lifetime data in reliability and medical studies. It is also models phenomena with increasing failure rate. However, the $L E$ distribution does not provide a reasonable parametric fit for modeling phenomenon with decreasing, non linear increasing, or non-monotone failure rates such as the bathtub shape, which are common in firm ware reliability modeling, biological studies, see Lai et al. (2001) and Zhang et al. (2005).

A new generalization of the linear exponential distribution is generalized linear exponential $(G L E)$ distribution. This distribution is important since it contains as special sub-models some widely well known distributions. It also provides more flexibility to analyze complex real data sets. (see Mahmoud and Alam (2010)).

A random variable $X$ is said to have the generalized linear exponential distribution with three parameters $\alpha, \beta$ and $\theta$, if it has the cumulative distribution function

$$
G(x, \alpha, \beta, \theta)=1-e^{-\left(\alpha x+\frac{\beta}{2} x^{2}\right)^{\theta}}, x>0, \alpha, \beta, \theta>0,
$$

and the corresponding probability density function (pdf) is given by

$$
\begin{aligned}
g(x, \alpha, \beta, \theta)= & \theta(\alpha+\beta x)\left(\alpha x+\frac{\beta}{2} x^{2}\right)^{\theta-1} e^{-\left(\alpha x+\frac{\beta}{2} x^{2}\right)^{\theta}} \\
& x>0, \alpha, \beta, \theta>0 .
\end{aligned}
$$

The quality of the procedures used in statistical analysis depends heavily on the assumed probability model or distributions. Because of this, considerable effort over the years has been expended in the development of large classes of standard probability distributions along with relevant statistical methodologies. In fact, the statistics literature is filled with hundreds of continuous univariate distributions. However, in recent years, applications from the environmental, financial, biomedical sciences, engineering among others, have further shown that data sets following the classical distributions are more often the exception rather than the reality. Since there is a clear need for extended forms of these distributions a significant progress has been made toward the generalization of some wellknown distributions and their successful application to problems in areas such as engineering, finance, economics and biomedical sciences, among others.

In this article we use transmutation map approach suggested by Shaw and Buckley (2007) to define a new model which generalizes the generalized linear exponential Distribution. We will call the generalized distribution as the transmuted generalized linear exponential distribution $\left(T_{G L E D}\right)$ distribution. According to the Quadratic Rank Transmutation Map,(QRTM), approach the cumulative distribution function (cdf) satisfy the relationship

$$
F_{2}(x)=(1+\lambda) F_{1}(x)-\lambda F_{1}(x)^{2}
$$

which on differentiation yields,

$$
f_{2}(x)=f_{1}(x)\left[(1+\lambda)-2 \lambda F_{1}(x)\right]
$$

where $f_{1}(x)$ and $f_{2}(x)$ are the corresponding pdfs associated with cdf $F_{1}(x)$ and $F_{2}(x)$ respectively and $-1 \leq \lambda \leq 1$. An extensive information about the quadratic rank transmutation map is given in Shaw and Buckley (2007).

We will use the above formulation for a pair of distributions $F(x)$ and $G(x)$ where $G(x)$ is a sub-model of $F(x)$. Therefore, a random variable $X$ is said to have a transmuted probability distribution with cdf $F(x)$ if

$$
F(x)=(1+\lambda) G(x)-\lambda G(x)^{2},|\lambda| \leq 1,
$$


where $G(x)$ is the cdf of the base distribution. Observe that at $\lambda$ $=0$ we have the distribution of the base random variable.

Many authors dealing with the generalization of some well- known distributions . Aryal and Tsokos (2009) defined the transmuted generalized extreme value distribution and they studied some basic mathematical characteristics of transmuted Gumbel probability distribution and it has been observed that the transmuted Gumbel can be used to model climate data. Also Aryal and Tsokos (2011) presented a new generalization of Weibull distribution called the transmuted Weibull distribution . Recently, Aryal (2013) proposed and studied the various structural properties of the transmuted LogLogistic distribution. and Muhammad khan and King (2013) introduced the transmuted modified Weibull distribution which extended recent development on transmuted Weibull distribution by Aryal et al. (2011). and they studied the mathematical properties and maximum likelihood estimation of the unknown parameters.In the present study we will provide mathematical formulation of the transmuted generalized linear exponential distribution $\left(T_{G L E D}\right)$ distribution and some of its properties.

Merovci and Elbatal (2013) introduce a new lifetime distribution by transmuted and compounding Lindley and geometric distributions named transmuted Lindley geometric distribution. They derive expansions for moments and for the moment generating function. The estimation of parameters is approached by the method of maximum likelihood, also the information matrix is derived. An application of the transmuted Lindley geometric distribution to real data. Elbatal (2013) proposed a functional composition of the cumulative distribution function of one probability distribution with the inverse cumulative distribution function of another is called the transmutation map.He used the quadratic rank transmutation map (QRTM) in order to generate a flexible family of probability distributions taking modified inverse weibull distribution as the base value distribution by introducing a new parameter that would offer more distributional flexibility. It will be shown that the analytical results are applicable to model real world data. Elbatal and Aryal (2013) presented the transmuted additive Weibull distribution, that extends the additive Weibull distribution and some other distributions they used the quadratic rank transmutation map (QRTM) proposed by Shaw \& Buckley( 2007) in order to generate the transmuted additive Weibull distribution. Various structural properties of the new distribution including the explicit expressions for the moments,random number generation and order statistics are derived. Maximum likelihood estimation of the unknown parameters of the new model for complete sample is also discussed. It will be shown that the analytical results are applicable to model real world data. The rest of the paper is organized as follows. In Section 2 we demonstrate transmuted probability distribution, and we present the flexibility of the subject distribution and some special sub-models. The reliability functions of the subject model are given in Section 3. In Section 4 we studied the statistical properties include quantile functions, moments, moment generating function . The minimum , maximum and median order statistics models are discussed in Section 5. Finally, In Section 6 we demonstrate the maximum likelihood estimates and the asymptotic confidence intervals of the unknown parameters. Finally, some lifetime data sets are used to illustrate that the generalized linear exponential distribution (TGLED) can used for the data under analysis, comparing with some known distributions.

\section{TRANSMUTED GENERALIZED LINEAR EXPONENTIAL DISTRIBUTION}

In this section we studied the transmuted generalized linear exponential distribution $\left(T_{G L E D}\right)$ and the sub-models of this distribution. Now using ?? and ?? we have the cdf of transmuted generalized linear exponential distribution

$$
\begin{aligned}
F_{T G L E} & =(1+\lambda)\left(1-e^{-\left(\alpha x+\frac{\beta}{2} x^{2}\right)^{\theta}}\right)-\lambda\left(1-e^{-\left(\alpha x+\frac{\beta}{2} x^{2}\right)^{\theta}}\right)^{2}, \\
& =\left[1-e^{-\left(\alpha x+\frac{\beta}{2} x^{2}\right)^{\theta}}\right]\left[1+\lambda e^{-\left(\alpha x+\frac{\beta}{2} x^{2}\right)^{\theta}}\right]
\end{aligned}
$$

where $\alpha, \beta$ are the scale parameters, $\theta$ is shape parameter representing the different patterns of the transmuted generalized linear exponential distribution and $\lambda$ is the transmuted parameter. The restrictions in equation (6) on the values of $\alpha, \beta, \theta$ and $\lambda$ are always the same. The probability density function (pdf) of the transmuted generalized linear exponential distribution is given by

$$
\begin{aligned}
f_{\text {TGIE }}(x)= & \theta(\alpha+\beta x)\left(\left(\alpha x+\frac{\beta}{2} x^{2}\right)^{\theta-1} e^{-\left(\alpha x+\frac{\beta}{2} x^{2}\right)^{\theta}} \times\right. \\
& {\left[1-\lambda+2 \lambda e^{-\left(\alpha x+\frac{\beta}{2} x^{2}\right)^{\theta}}\right] . }
\end{aligned}
$$

The transmuted generalized linear exponential distribution is very flexible model that approaches to different distributions when its parameters are changed.

(i) If $\lambda=0$ we get the generalized linear exponential distribution $\operatorname{GLED}(\alpha, \beta, \theta)$.

(ii) If $\alpha=\frac{1}{\sigma}, \beta=0$ we get the transmuted Weibull distribution $T_{W D}(\lambda, \sigma, \theta)$.

(iii) If $\lambda=\beta=0, \alpha=\frac{1}{\sigma}$ we get theWeibull distribution $W(\sigma, \theta)$.

(iv) If $\theta=1$ we get the transmuted linear exponential distribution $T_{L E D}(\lambda, \alpha, \beta)$

(v) If $\theta=1, \alpha=0$ we get the transmuted Rayleigh distribution $T_{R D}(\lambda, \beta)$

(vi) If $\theta=1, \alpha=\lambda=0$ we get the Rayleigh distribution $R D$ $(\beta)$.

Figure 1 shows both cases of the shape parameter $\theta>1$ and $\theta \leq 1$.

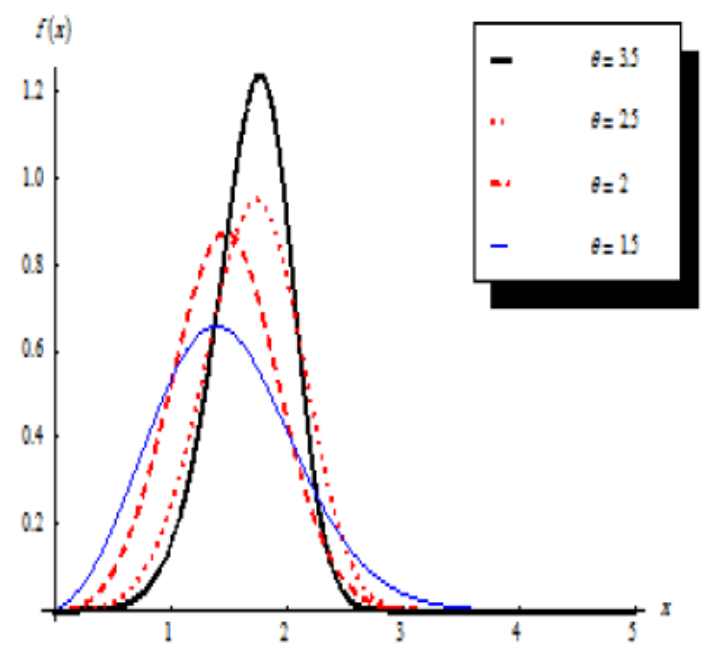




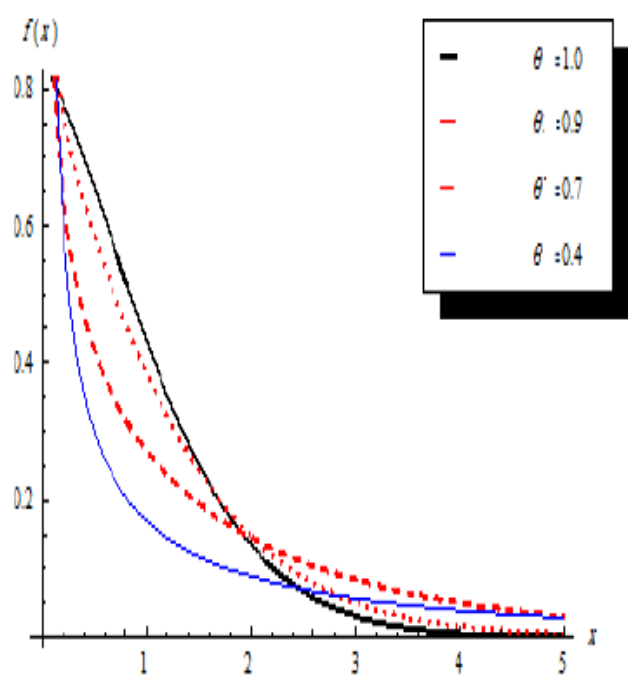

Fig. 1. Effect of shape parameter $\theta$ on the TGLED PDF

\section{RELIABILITY ANALYSIS}

The transmuted generalized linear exponential distribution can be a useful characterization of life time data analysis. The reliability function $(R F)$ of the transmuted generalized linear exponential distribution is denoted by $R_{T_{G L E D}}(x)$ also known as the survivor function and is defined as

$$
\begin{aligned}
& R_{T_{G L E D}}(x) \\
= & 1-F_{T_{G L E D}}(x) \\
= & 1-\left[1-e^{-\left(\alpha x+\frac{\beta}{2} x^{2}\right)^{\theta}}\right]\left[1+\lambda e^{-\left(\alpha x+\frac{\beta}{2} x^{2}\right)^{\theta}}\right] .
\end{aligned}
$$

Figure 2 (a) and (b) represent the CDF and RF respectively for different values of shape parameter $\theta$.

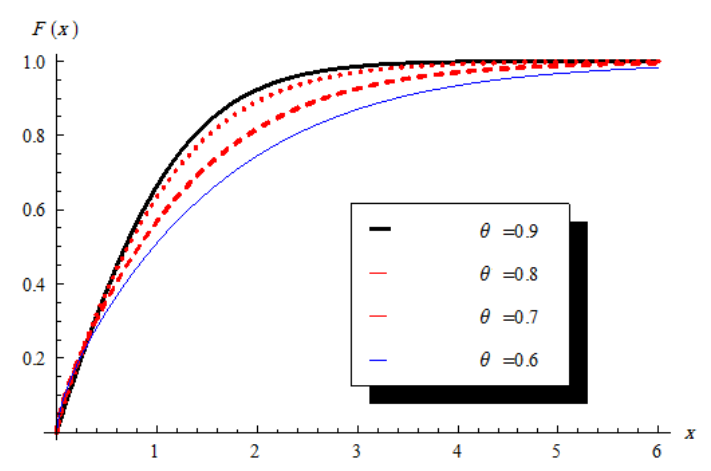

Fig. 2(a) $\mathrm{CDF}$

Fig. 2(b) RF

It is important to note that $R_{T_{G L E D}}(x)+F_{T_{G L E D}}(x)=1$. One of the characteristic in reliability analysis is the hazard rate function (HRF) defined by

$$
h_{\text {TGIE }}(x)
$$

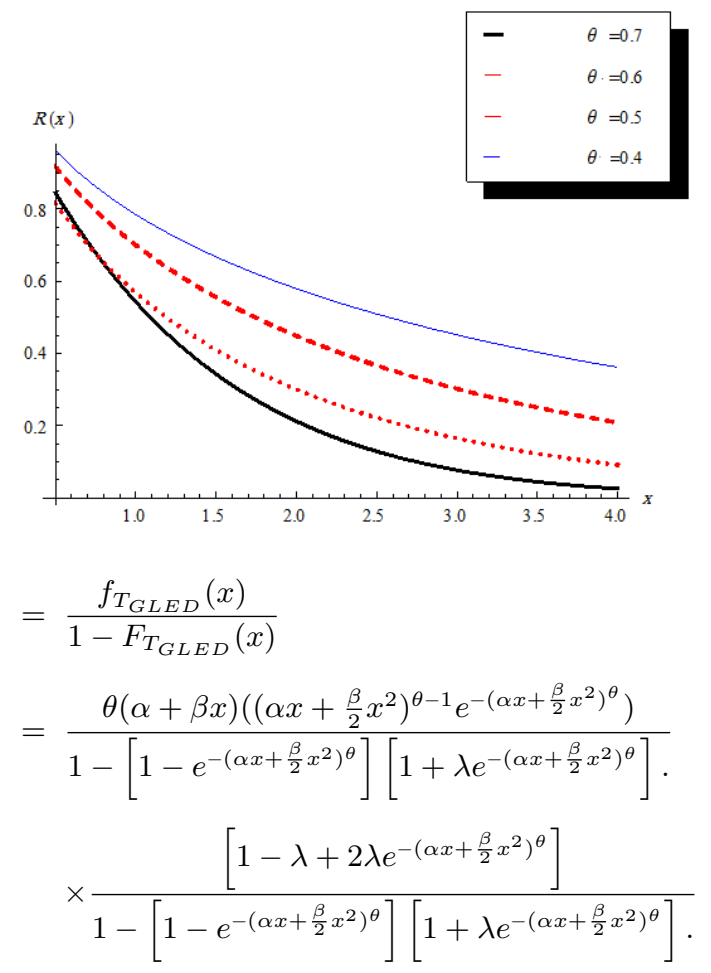

It is important to note that the units for $h_{T G I E}(x)$ is the probability of failure per unit of time, distance or cycles. These failure rates are defined with different choices of parameters. The cumulative hazard function of the transmuted generalized inverted exponential distribution is denoted by $H_{T G I E}(x)$ and is defined as

$$
H_{T G I E}(x)=-\ln \left|\left[1-e^{-\left(\alpha x+\frac{\beta}{2} x^{2}\right)^{\theta}}\right]\left[1+\lambda e^{-\left(\alpha x+\frac{\beta}{2} x^{2}\right)^{\theta}}\right]\right|
$$

It is important to note that the units for $H_{T G I E}(x)$ is the cumulative probability of failure per unit of time, distance or cycles. we can show that . For all choice of parameters the distribution has the decreasing patterns of cumulative instantaneous failure rates. Figure 3 (a),(b) and (c) represent the hazard rate function (HRF) with different values of shape parameter $\theta$. 


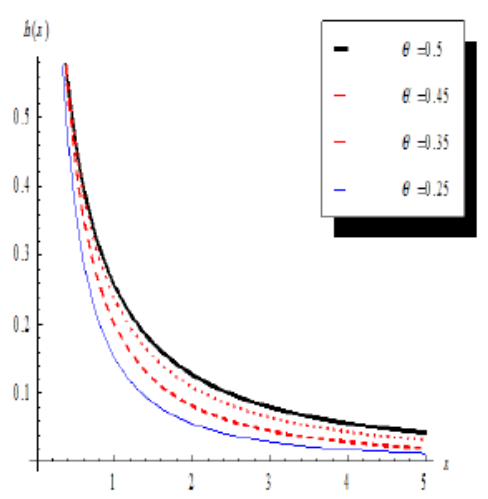

Fig. 3(a)

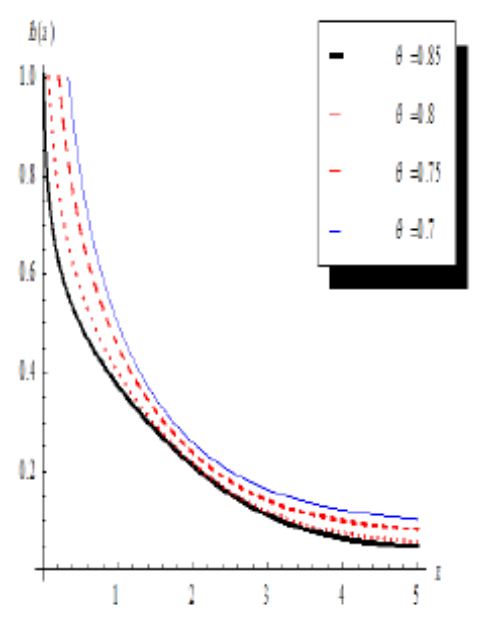

Fig. 3(b)

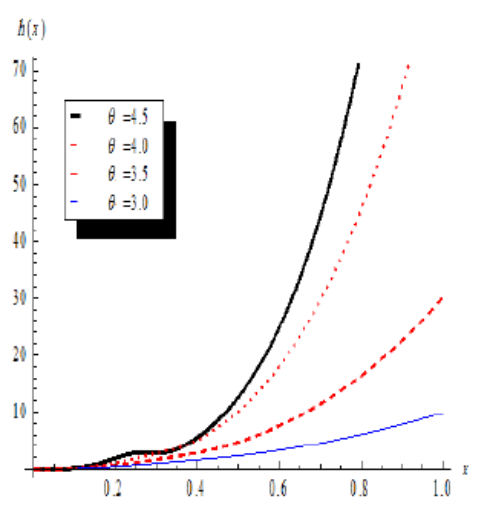

Fig. 3(c)
Fig. (3) Effect of shape parameter $\theta$ on the hazard rate function (HRF) of the TGLED.

THEOREM 1. The hazard rate function of the transmuted generalized linear exponential distribution has the following properties

(i) If $\lambda=0$ we get the failure rate is same as the $G L E D(\alpha, \beta, \theta)$.

(ii) If $\alpha=\frac{1}{\sigma}, \beta=0$ we get the the failure rate is same as the $T_{W D}(\lambda, \sigma, \theta)$.

(iii) If $\lambda=\beta=0, \alpha=\frac{1}{\sigma}$ we get the the failure rate is same as the $W(\sigma, \theta)$.

(iv) If $\theta=1$ we get the the failure rate is same as the $T_{L E D}(\lambda, \alpha, \beta)$.

PROOF. The hazard function (HF) of the transmuted generalized linear exponential distribution is given in equation (9) has the special cases with different choice of parameters:

(i) If $\lambda=0$ we get the failure rate is same as the $\operatorname{GLED}(\alpha, \beta, \theta)$

$$
h_{G L E D}=\theta(\alpha+\beta x)\left(\left(\alpha x+\frac{\beta}{2} x^{2}\right)^{\theta-1}\right.
$$

(ii) If $\alpha=\frac{1}{\sigma}, \beta=0$ we get the the failure rate is same as the $T_{W D}(\lambda, \sigma, \theta)$.

$$
h_{T_{W D}}(x)=\frac{\frac{\theta x}{\sigma}\left(\frac{x}{\sigma}\right)^{\theta-1} e^{-\left(\frac{x}{\sigma}\right)^{\theta}}\left[1-\lambda+2 \lambda e^{-\left(\frac{x}{\sigma}\right)^{\theta}}\right]}{1-\left[1-e^{-\left(\frac{x}{\sigma}\right)^{\theta}}\right]\left[1+\lambda e^{-\left(\frac{x}{\sigma}\right)^{\theta}}\right] .} .
$$

(iii) If $\lambda=\beta=0, \alpha=\frac{1}{\sigma}$ we get the the failure rate is same as the $W(\sigma, \theta)$

$$
h_{W D}(x)=\frac{\frac{\theta x}{\sigma}\left(\frac{x}{\sigma}\right)^{\theta-1} e^{-\left(\frac{x}{\sigma}\right)^{\theta}}}{1-\left[1-e^{-\left(\frac{x}{\sigma}\right)^{\theta}}\right]}
$$

(iv) If $\theta=1$ we get the the failure rate is same as the $T_{L E D}(\lambda, \alpha, \beta)$

$$
h_{T L E D}(x)=\frac{(\alpha+\beta x) e^{-\left(\alpha x+\frac{\beta}{2} x^{2}\right)}\left[1-\lambda+2 \lambda e^{-\left(\alpha x+\frac{\beta}{2} x^{2}\right)}\right]}{1-\left[1-e^{-\left(\alpha x+\frac{\beta}{2} x^{2}\right)}\right]\left[1+\lambda e^{-\left(\alpha x+\frac{\beta}{2} x^{2}\right)}\right] .}
$$

\section{STATISTICAL PROPERTIES}

This section is devoted to study statistical properties for the transmuted generalized linear exponential, specifically Quantile function ,median, moments, moment generating function.

\subsection{Quantile and Median}

The quantile $x_{q}$ of the $T_{G L E D}(\alpha, \beta, \theta, \lambda, x)$ is real solution of the following equation

$$
x_{q}=\frac{-\alpha+\sqrt{\alpha^{2}+2 \beta\left[-\ln \frac{(\lambda-1)+\sqrt{(\lambda+1)^{2}-4 \lambda q}}{2 \lambda}\right]^{\frac{1}{\theta}}}}{\beta}
$$

The above equation has no closed form solution in $x_{q}$, so we have to use a numerical technique such as a Newton- Raphson method to get the quantile. If we put $q=0.5$ in equation (11) one gets the median of $T_{G L E D}(\alpha, \beta, \theta, \lambda, x)$ 


\subsection{Random Number Generation}

The random number generation as $x$ of the $T_{G L E D}(\alpha, \beta, \theta, \lambda, x)$ is defined by the following relation

$$
\left[1-e^{-\left(\alpha x+\frac{\beta}{2} x^{2}\right)}\right]\left[1+\lambda e^{-\left(\alpha x+\frac{\beta}{2} x^{2}\right)}\right]=\varphi \text { where } \varphi \sim U(0,1)
$$

thus

$$
x=\frac{-\alpha+\sqrt{\alpha^{2}+2 \beta\left[-\ln \frac{(\lambda-1)+\sqrt{(\lambda+1)^{2}-4 \lambda \varphi}}{2 \lambda}\right]^{\frac{1}{\theta}}}}{\beta} .
$$

\subsection{Moments}

In this subsection, we derive the $r_{t h}$ moments and moment generating function $M_{X}(t)$ of the $T G L E$. The following theorem gives the $r_{t h}$ moment $\left(\mu_{r}\right)$ of the $T_{G L E D}(\alpha, \beta, \theta, \lambda, x$.

THEOREM 2. If $X$ has TGLE, then the $r_{\text {th }}$ moment of $X, r=$ $1,2, \ldots$.has the following form:

$$
\begin{aligned}
\mu_{r}^{\prime}= & \sum_{i=0}^{r} \sum_{j=0}^{\infty}(-1)^{i+j}\left(\begin{array}{c}
r \\
i
\end{array}\right)\left(\begin{array}{c}
\frac{r-i}{2} \\
j
\end{array}\right) 2^{\frac{r-i}{2}-j} \times \\
& \alpha^{i+2 j} \frac{1}{\beta^{\frac{r+i}{2}+j}}\left\{(1-\lambda) \Gamma\left(\frac{r-i}{2 \theta}-\frac{j}{\theta}+1\right)\right. \\
& \left.+\lambda 2^{-\left(\frac{r+2 \theta-i}{2 \theta}\right)+\frac{j}{\theta}} \Gamma\left(\frac{r-i}{2 \theta}-\frac{j}{\theta}+1\right)\right\} .
\end{aligned}
$$

PROOF. We start with the well known definition of the $r_{t h}$ moment of the random variable $X$ with pdf of transmuted generalized linear exponential given by

$$
\begin{aligned}
\mu_{r}^{\prime}= & E\left(X^{r}\right) \\
= & \int_{0}^{\infty} x^{r} f_{T G L E}(x) d x \\
= & \left\{(1-\lambda) \int_{0}^{\infty} x^{r} \theta(\alpha+\beta x)\left(\alpha x+\frac{\beta}{2} x^{2}\right)^{\theta-1} e^{-\left(\alpha x+\frac{\beta}{2} x^{2}\right)^{\theta}}\right. \\
& \left.+2 \lambda \int_{0}^{\infty} \theta x^{r}(\alpha+\beta x)\left(\alpha x+\frac{\beta}{2} x^{2}\right)^{\theta-1} e^{-2\left(\alpha x+\frac{\beta}{2} x^{2}\right)^{\theta}}\right\} \\
& \times d x
\end{aligned}
$$

Now we define the following substitution $y=\left(\alpha x+\frac{\beta}{2} x^{2}\right)^{\theta}$ this implies that $d y=\theta\left(\alpha x+\frac{\beta}{2} x^{2}\right)^{\theta-1}(\alpha+\beta x) d x$. Clearly,

$$
x=\frac{-\alpha+\sqrt{\alpha^{2}+2 \beta y^{\frac{1}{\theta}}}}{\beta}
$$

Thus

$$
\begin{aligned}
\mu_{r}^{\prime}= & (1-\lambda)\left\{\int_{0}^{\infty}\left[\frac{-\alpha+\sqrt{\alpha^{2}+2 \beta y^{\frac{1}{\theta}}}}{\beta}\right]^{r} e^{-y}\right. \\
& \left.+2 \lambda\left[\frac{-\alpha+\sqrt{\alpha^{2}+2 \beta y^{\frac{1}{\theta}}}}{\beta}\right]^{r} e^{-2 y}\right\} d y,
\end{aligned}
$$

by using the binomial series expansion of $\left[-\alpha+\sqrt{\alpha^{2}+2 \beta y^{\frac{1}{\theta}}}\right]^{r}$ we get

$$
\left[-\alpha+\sqrt{\alpha^{2}+2 \beta y^{\frac{1}{\theta}}}\right]^{r}=\sum_{i=0}^{r}(-1)^{i}\left(\begin{array}{c}
r \\
i
\end{array}\right) \alpha^{i}\left(\alpha^{2}+2 \beta y^{\frac{1}{\theta}}\right)^{\frac{r-i}{2}},
$$

and

$$
\left(\alpha^{2}+2 \beta y^{\frac{1}{\theta}}\right)^{\frac{r-i}{2}}=(2 \beta)^{\frac{r-i}{2}} y^{\frac{r-i}{2 \theta}} \sum_{j=0}^{\infty}(-1)^{j}\left(\begin{array}{c}
\frac{r-i}{2} \\
j
\end{array}\right)\left(\frac{\alpha^{2}}{2 \beta y^{\frac{1}{\theta}}}\right)^{j},
$$

substituting from 15 and 16 into 14 , we have the following

$$
\begin{aligned}
\mu_{r}^{\prime}= & \sum_{i=0}^{r} \sum_{j=0}^{\infty}(-1)^{i+j}\left(\begin{array}{c}
r \\
i
\end{array}\right)\left(\begin{array}{c}
\frac{r-i}{2} \\
j
\end{array}\right) 2^{\frac{r-i}{2}-j} \alpha^{i+2 j} \frac{1}{\beta^{\frac{r+i}{2}+j}} \times \\
& \left\{\int_{0}^{\infty} y^{\frac{r-i}{2 \theta}-\frac{j}{\theta}} e^{-y} \frac{2 \lambda}{\beta^{r}} \int_{0}^{\infty} y^{\frac{r-i}{2 \theta}-\frac{j}{\theta}} e^{-2 y}\right\} d y \\
= & \sum_{i=0}^{r} \sum_{j=0}^{\infty}(-1)^{i+j}\left(\begin{array}{c}
r \\
i
\end{array}\right)\left(\begin{array}{c}
\frac{r-i}{2} \\
j
\end{array}\right) 2^{\frac{r-i}{2}-j} \alpha^{i+2 j} \frac{1}{\beta^{\frac{r+i}{2}+j}} \times \\
& \left\{(1-\lambda) \Gamma\left(\frac{r-i}{2 \theta}-\frac{j}{\theta}+1\right)\right. \\
& \left.+\lambda 2^{-\left(\frac{r+2 \theta-i}{2 \theta}\right)+\frac{j}{\theta}} \Gamma\left(\frac{r-i}{2 \theta}-\frac{j}{\theta}+1\right)\right\}
\end{aligned}
$$

therefore

$$
\mu_{r}^{\prime}=C_{i, j} \Gamma\left(\frac{r-i}{2 \theta}-\frac{j}{\theta}+1\right)\left[1-\lambda+\lambda\left(\frac{1}{2}\right)^{\frac{r-i}{2 \theta}-\frac{j}{\theta}+1}\right]
$$

where

$$
C_{i, j}=\sum_{i=0}^{r} \sum_{j=0}^{\infty}(-1)^{i+j}\left(\begin{array}{c}
r \\
i
\end{array}\right)\left(\begin{array}{c}
\frac{r-i}{2} \\
j
\end{array}\right) 2^{\frac{r-i}{2}-j} \alpha^{i+2 j} \frac{1}{\beta^{\frac{3 r+i}{2}+j}}
$$

which completes the proof .

THEOREM 3. If $X$ has TGLE, then the moment generating function $M_{X}(t)$ has the following form

$$
\begin{aligned}
M_{X}(t)= & C_{i, j} \sum_{r=0}^{\infty} \frac{t^{r}}{r !} \Gamma\left(\frac{r-i}{2 \theta}-\frac{j}{\theta}+1\right) \times \\
& {\left[1-\lambda+\lambda\left(\frac{1}{2}\right)^{\frac{r-i}{2 \theta}-\frac{j}{\theta}+1}\right] }
\end{aligned}
$$

Proof. We start with the well known definition of the moment generating function given by

$$
\begin{aligned}
M_{X}(t) & =E\left(e^{t x}\right) \\
& =\int_{0}^{\infty} e^{t x} f_{T G L E}(x) d x \\
& =\int_{0}^{\infty} \sum_{r=0}^{\infty} \frac{t^{r} x^{r}}{r !} f_{T G L E}(x) d x \\
& =\sum_{r=0}^{\infty} \frac{t^{r}}{r !} \mu_{r}^{\prime}
\end{aligned}
$$




$$
\begin{gathered}
=C_{i, j} \sum_{r=0}^{\infty} \frac{t^{r}}{r !} \Gamma\left(\frac{r-i}{2 \theta}-\frac{j}{\theta}+1\right) \times \\
{\left[1-\lambda+\lambda\left(\frac{1}{2}\right)^{\frac{r-i}{2 \theta}-\frac{j}{\theta}+1}\right]}
\end{gathered}
$$

which completes the proof.

\section{ORDER STATISTICS}

The order statistics have many applications in reliability and life testing. The order statistics arise in the study of reliability of a system. Let $X_{1}, X_{2}, \ldots, X_{n}$ be a simple random sample from $T_{G L E D}$ $(\alpha, \beta, \theta, \lambda, x)$ with cumulative distribution function and probability density function as in 6 and 7 , respectively. Let $X_{(1: n)}$ $\leq X_{(2: n)} \leq \ldots \leq X_{(n: n)}$ denote the order statistics obtained from this sample. In reliability literature, $X_{(i: n)}$ denote the lifetime of an $(n-i+1)-$ out - of $-n$ system which consists of $n$ independent and identically components. Then the pdf of $X_{(i: n)}, 1 \leq i \leq n$ is given by

$$
f_{i:: n}(x)=\frac{1}{\beta(i, n-i+1)}[F(x, \Phi)]^{i-1}[1-F(x, \Phi)]^{n-i} f(x, \Phi)
$$

where $\Phi=(\alpha, \beta, \theta, \lambda$,$) also, the joint pdf of X_{(i: n)}, X_{(j: n)}$ and $1 \leq i \leq j \leq n$ is

$$
\begin{aligned}
f_{i: j: n}\left(x_{i}, x_{j}\right)= & C\left[F\left(x_{i}\right)\right]^{i-1}\left[F\left(x_{j}\right)-F\left(x_{i}\right)\right]^{j-i-1} \times \\
& {\left[1-F\left(x_{j}\right)\right]^{n-j} f\left(x_{i}\right) f\left(x_{j}\right) }
\end{aligned}
$$

where $\mathrm{C}$

$$
C=\frac{n !}{(i-1) !(j-i-1) !(n-j) !}
$$

We defined the first order statistics $X_{(1)}=\operatorname{Min}\left(X_{1}, X_{2}, \ldots, X_{n}\right)$, the the last order statistics as $X_{(n)}=\operatorname{Max}\left(X_{1}, X_{2}, \ldots, X_{n}\right)$ and median order $X_{m+1}$.

\subsection{Distribution of Minimum, Maximum and Median}

Let $X_{1}, X_{2}, \ldots, X_{n}$ be independently identically distributed order random variables from the transmuted generalized linear exponential distribution having first, last and median order probability density function are given by the following

$$
\begin{aligned}
& f_{1: n}(x) \\
= & n[1-F(x, \Phi)]^{n-1} f(x, \Phi) \\
= & n\left(1-\left[1-e^{-\left(\alpha x_{(1)}+\frac{\beta}{2} x_{(1)}^{2}\right)^{\theta}}\right]\left[1+\lambda e^{-\left(\alpha x_{(1)}+\frac{\beta}{2} x_{(1)}^{2}\right)^{\theta}}\right]\right)^{n-1} \\
\times & {\left[\theta\left(\alpha+\beta x_{(1)}\right)\left(\alpha x_{(1)}+\frac{\beta}{2} x_{(1)}^{2}\right)^{\theta-1} e^{-\left(\alpha x_{(1)}+\frac{\beta}{2} x_{(1)}^{2}\right)^{\theta}}\right] } \\
& \times\left[1-\lambda+2 \lambda e^{-\left(\alpha x_{(1)}+\frac{\beta}{2} x_{(1)}^{2}\right)^{\theta}}\right]
\end{aligned}
$$

$$
f_{n: n}(x)
$$

$$
\begin{aligned}
= & \left.n\left[F\left(x_{(n)}, \Phi\right)\right]^{n-1} f\left(x_{(n)}\right), \Phi\right) \\
= & n\left\{\left[1-e^{-\left(\alpha x_{(n)}+\frac{\beta}{2} x_{(n)}^{2}\right)^{\theta}}\right]\left[1+\lambda e^{-\left(\alpha x_{(n)}+\frac{\beta}{2} x_{(n)}^{2}\right)^{\theta}}\right]\right\}^{n-1} \\
& \times\left[\theta\left(\alpha+\beta x_{(n)}\right)\left(\alpha x_{(n)}+\frac{\beta}{2} x_{(n)}^{2}\right)^{\theta-1} e^{-\left(\alpha x_{(n)}+\frac{\beta}{2} x_{(n)}^{2}\right)^{\theta}}\right] \\
& \times\left[1-\lambda+2 \lambda e^{-\left(\alpha x_{(n)}+\frac{\beta}{2} x_{(n)}^{2}\right)^{\theta}}\right]
\end{aligned}
$$

and

$$
\begin{aligned}
f_{m+1: n}(\widetilde{x})= & \frac{(2 m+1) !}{m ! m !}(F(\widetilde{x}))^{m}(1-F(\widetilde{x}))^{m} f(\widetilde{x}) \\
= & \frac{(2 m+1) !}{m ! m !}\left\{\left[1-e^{-\left(\alpha x_{(m+1)}+\frac{\beta}{2} x_{(m+1)}^{2}\right)^{\theta}}\right]\right. \\
& \left.\times\left[1+\lambda e^{-\left(\alpha x_{(m+1)}+\frac{\beta}{2} x_{(m+1)}^{2}\right)^{\theta}}\right]\right\}^{m} \\
& \times\left\{\left(1-\left[1-e^{-\left(\alpha x_{(m+1)}+\frac{\beta}{2} x_{(m+1)}^{2}\right)^{\theta}}\right]\right.\right. \\
& \left.\left.\times\left[1+\lambda e^{\left.-e^{-(\alpha x}(m+1)+\frac{\beta}{2} x_{(m+1)}^{2}\right)^{\theta}}\right]\right)\right\}^{m} \\
& \times\left[\theta\left(\alpha+\beta x_{(m+1)}\right)\left(\alpha x_{(m+1)}+\frac{\beta}{2} x_{(m+1)}^{2}\right)^{\theta-1} \times\right. \\
& \left.e^{-\left(\alpha x_{(m+1)}+\frac{\beta}{2} x_{(m+1)}^{2}\right)^{\theta}}\right] \\
& \times\left[1-\lambda+2 \lambda e^{-\left(\alpha x_{(m+1)}+\frac{\beta}{2} x_{(m+1)}^{2}\right)^{\theta}}\right]
\end{aligned}
$$

\subsection{Joint Distribution of the $\mathbf{i}_{t h}$ and $\mathbf{j}_{t h}$ order Statistics}

The joint distribution of the the $i_{t h}$ and $j_{t h}$ order Statistics from transmuted generalized linear exponential distribution is

$$
\begin{aligned}
& f_{i: j: n}\left(x_{i}, x_{j}\right) \\
= & C\left[F\left(x_{i}\right)\right]^{i-1}\left[F\left(x_{j}\right)-F\left(x_{i}\right)\right]^{j-i-1}\left[1-F\left(x_{j}\right)\right]^{n-j} f\left(x_{i}\right) f\left(x_{j}\right) \\
= & C\left\{\left[1-h_{(i)}\right]\left[1+\lambda h_{(i)}\right]\right\}^{i-1} \\
& \times\left\{\left[1-h_{(j)}\right]\left[1+\lambda h_{(j)}\right]-\left[1-h_{(i)}\right]\left[1+\lambda h_{(i)}\right]\right\}^{j-i-1} \\
& \times\left\{1-\left[1-h_{(j)}\right]\left[1+\lambda h_{(j)}\right]\right\}^{n-j} \\
& \times\left[\theta\left(\alpha+\beta x_{(i)}\right)\left(\alpha x_{(i)}+\frac{\beta}{2} x_{(i)}^{2}\right)^{\theta-1} h_{(i)}\right]\left[1-\lambda+2 \lambda h_{(i)}\right] \\
& \times\left[\theta\left(\alpha+\beta x_{(j)}\right)\left(\alpha x_{(j)}+\frac{\beta}{2} x_{(j)}^{2}\right)^{\theta-1} h_{(j)}\right]\left[1-\lambda+2 \lambda h_{(j)}\right]
\end{aligned}
$$

where

$$
h_{(i)}=e^{-\left(\alpha x_{(i)}+\frac{\beta}{2} x_{(i)}^{2}\right)^{\theta}}
$$


special case if $i=1$ and $j=n$ we get the joint distribution of the minimum and maximum of order statistics

$$
\begin{aligned}
& f_{1:: n: n}\left(x_{i}, x_{j}\right) \\
= & n(n-1)\left[F\left(x_{(n)}\right)-F\left(x_{(1)}\right)\right]^{n-2} f\left(x_{(1)}\right) f\left(x_{(n)}\right) \\
= & n(n-1)\left\{\left[1-h_{(n)}\right]\left[1+\lambda h_{(n)}\right]-\left[1-h_{(1)}\right]\left[1+\lambda h_{(1)}\right]\right\}^{n-2} \\
& \times\left[\theta\left(\alpha+\beta x_{(1)}\right)\left(\alpha x_{(1)}+\frac{\beta}{2} x_{(1)}^{2}\right)^{\theta-1} h_{(1)}\right]\left[1-\lambda+2 \lambda h_{(1)}\right] \\
& \times\left[\theta\left(\alpha+\beta x_{(n)}\right)\left(\alpha x_{(n)}+\frac{\beta}{2} x_{(n)}^{2}\right)^{\theta-1} h_{(n)}\right]\left[1-\lambda+2 \lambda h_{(n)}\right] .
\end{aligned}
$$

\section{ESTIMATION AND INFERENCE}

In this section we discuss the maximum likelihood estimators (MLE's) and inference for the $T_{G L E}(\alpha, \beta, \theta, \lambda, x)$. distribution. Let $X_{1}, \ldots, X_{n}$ be a random sample of size $n$ from $T_{G L E}(\alpha, \beta, \theta, \lambda, x)$ then the likelihood function can be written as

$$
\begin{aligned}
& L\left(\theta, \alpha, \lambda, x_{(i)}\right) \\
= & \prod_{i=1}^{n} f\left(x_{i}, \alpha, \beta, \theta, \lambda\right) d x \\
= & \prod_{i=1}^{n} \theta\left(\alpha+\beta x_{(i)}\right)\left(\alpha x_{(i)}+\frac{\beta}{2} x_{(i)}^{2}\right)^{\theta-1} e^{-\left(\alpha x_{(i)}+\frac{\beta}{2} x_{(i)}^{2}\right)^{\theta}} \\
& \times\left[1-\lambda+2 \lambda e^{-\left(\alpha x_{(i)}+\frac{\beta}{2} x_{(i)}^{2} \theta^{\theta}\right.}\right]
\end{aligned}
$$

By accumulation taking logarithm of equation 27, and the loglikelihood function can be written as

$$
\begin{aligned}
\log L= & n \ln \theta+\sum_{i=1}^{n} \ln \left(\alpha+\beta x_{i}\right) \\
& +(\theta-1) \sum_{i=1}^{n} \ln \left(\alpha x_{i}+\frac{\beta}{2} x_{i}^{2}\right)-\sum_{i=1}^{n}\left(\alpha x_{i}+\frac{\beta}{2} x_{i}^{2}\right)^{\theta} \\
& +\sum_{i=1}^{n} \ln \left[1-\lambda+2 \lambda e^{-\left(\alpha x_{i}+\frac{\beta}{2} x_{i}^{2}\right)^{\theta}}\right]
\end{aligned}
$$

Differentiating equation 28, with respect to $\alpha, \beta, \theta$ and $\lambda$ then the normal equations become

$$
\begin{aligned}
\frac{\partial \log L}{\partial \alpha}= & \sum_{i=1}^{n} \frac{1}{\left(\alpha+\beta x_{i}\right)}+(\theta-1) \sum_{i=1}^{n} \frac{x_{i}}{\left(\alpha x_{i}+\frac{\beta}{2} x_{i}^{2}\right)} \\
& -\theta \sum_{i=1}^{n} x_{i}\left(\alpha x_{i}+\frac{\beta}{2} x_{i}^{2}\right)^{\theta-1}
\end{aligned}
$$

$$
-2 \sum_{i=1}^{n} \frac{\theta \lambda x_{i} e^{-\left(\alpha x_{i}+\frac{\beta}{2} x_{i}^{2}\right)^{\theta}}\left(\alpha x_{i}+\frac{\beta}{2} x_{i}^{2}\right)^{\theta-1}}{\left[1-\lambda+2 \lambda e^{-\left(\alpha x_{i}+\frac{\beta}{2} x_{i}^{2}\right)^{\theta}}\right]}
$$

$$
\begin{aligned}
\frac{\partial \log L}{\partial \beta}= & \sum_{i=1}^{n} \frac{x_{i}}{\left(\alpha x_{i}+\frac{\beta}{2} x_{i}^{2}\right)}+\frac{(\theta-1)}{2} \sum_{i=1}^{n} \frac{x_{i}^{2}}{\left(\alpha x_{i}+\frac{\beta}{2} x_{i}^{2}\right)} \\
& -\frac{\theta}{2} \sum_{i=1}^{n} x_{i}^{2}\left(\alpha x_{i}+\frac{\beta}{2} x_{i}^{2}\right)^{\theta-1} \\
& -\sum_{i=1}^{n} \frac{\theta \lambda x_{i}^{2} e^{-\left(\alpha x_{i}+\frac{\beta}{2} x_{i}^{2}\right)^{\theta}}\left(\alpha x_{i}+\frac{\beta}{2} x_{i}^{2}\right)^{\theta-1}}{\left[1-\lambda+2 \lambda e^{-\left(\alpha x_{i}+\frac{\beta}{2} x_{i}^{2}\right)^{\theta}}\right]}
\end{aligned}
$$

$$
\begin{aligned}
& \frac{\partial \log L}{\partial \theta} \\
= & \frac{n}{\theta}+\sum_{i=1}^{n}\left(\alpha x_{i}+\frac{\beta}{2} x_{i}^{2}\right)-\sum_{i=1}^{n}\left(\alpha x_{i}+\frac{\beta}{2} x_{i}^{2}\right)^{\theta} \ln \left(\alpha x_{i}+\frac{\beta}{2} x_{i}^{2}\right) \\
& +2 \sum_{i=1}^{n} \frac{\lambda e^{-\left(\alpha x_{i}+\frac{\beta}{2} x_{i}^{2}\right)^{\theta}}\left(\alpha x_{i}+\frac{\beta}{2} x_{i}^{2}\right)^{\theta} \ln \left(\alpha x_{i}+\frac{\beta}{2} x_{i}^{2}\right)}{\left[1-\lambda+2 \lambda e^{-\left(\alpha x_{i}+\frac{\beta}{2} x_{i}^{2}\right)^{\theta}}\right]},
\end{aligned}
$$

and

$$
\frac{\partial \log L}{\partial \lambda}=\sum_{i=1}^{n} \frac{2 e^{-\left(\alpha x_{i}+\frac{\beta}{2} x_{i}^{2}\right)^{\theta}}-1}{\left[1-\lambda+2 \lambda e^{-\left(\alpha x_{i}+\frac{\beta}{2} x_{i}^{2}\right)^{\theta}}\right]} .
$$

We can find the estimates of the unknown parameters by maximum likelihood method by setting these above nonlinear system of equations 29 - 32 to zero and solve them simultaneously. These solutions will yield the ML estimators for $\widehat{\alpha}, \widehat{\beta}, \widehat{\theta}$, and $\widehat{\lambda}$, For the four parameters transmuted generalized linear exponential distribution $T_{G L E}(\alpha, \beta, \theta, \lambda, x)$ pdf, all the second order derivatives exist. Thus we have the inverse dispersion matrix is given by

$$
\left(\begin{array}{c}
\widehat{\alpha} \\
\widehat{\beta} \\
\widehat{\theta} \\
\widehat{\lambda}
\end{array}\right) \sim N\left[\left(\begin{array}{c}
\alpha \\
\beta \\
\theta \\
\lambda
\end{array}\right),\left(\begin{array}{|c|c|c|c|}
\widehat{V_{\alpha \alpha}} & \widehat{V_{\alpha \beta}} & \widehat{V_{\alpha \theta}} & \widehat{V_{\alpha \lambda}} \\
\hline \widehat{V_{\beta \alpha}} & \widehat{V_{\beta \beta}} & \widehat{V_{\beta \theta}} & \widehat{V_{\beta \lambda}} \\
\hline \widehat{V_{\theta \alpha}} & \widehat{V_{\theta \beta}} & \widehat{V_{\theta \theta}} & \widehat{V_{\theta \lambda}} \\
\hline \widehat{V_{\lambda \alpha}} & \widehat{V_{\lambda \beta}} & \widehat{V_{\lambda \theta}} & \widehat{V_{\lambda \lambda}} \\
\hline
\end{array}\right)\right]
$$

$$
V^{-1}=-E \begin{array}{|c|c|c|c||}
\hline V_{\alpha \alpha} & V_{\alpha \beta} & V_{\alpha \theta} & V_{\alpha \lambda} \\
\hline V_{\beta \alpha} & V_{\beta \beta} & V_{\beta \theta} & V_{\beta \lambda} \\
\hline V_{\theta \alpha} & V_{\theta \beta} & V_{\theta \theta} & V_{\theta \lambda} \\
\hline V_{\lambda \alpha} & V_{\lambda \beta} & V_{\lambda \theta} & V_{\lambda \lambda} \\
\hline
\end{array}
$$

where

$$
\begin{aligned}
& V_{\alpha \alpha}=\frac{\partial^{2} L}{\partial \alpha^{2}}, V_{\theta \theta}=\frac{\partial^{2} L}{\partial \theta^{2}}, V_{\lambda \lambda}=\frac{\partial^{2} L}{\partial \lambda^{2}}, V_{\beta \beta}=\frac{\partial^{2} L}{\partial \beta^{2}} \\
& V_{\lambda \alpha}=\frac{\partial^{2} L}{\partial \alpha \partial \lambda}, V_{\alpha \beta}=\frac{\partial^{2} L}{\partial \alpha \partial \beta}, V_{\alpha \theta}=\frac{\partial^{2} L}{\partial \alpha \partial \theta} .
\end{aligned}
$$

By solving this inverse dispersion matrix these solutions will yield asymptotic variance and covariances of these ML estimators for $\widehat{\alpha}$, 


\begin{tabular}{|c|c|c|c|c|c|}
\hline$T_{G L E D}(\alpha, \beta, \theta, \lambda)$ & $n$ & $M S E(\widehat{\alpha})$ & $M S E(\widehat{\beta})$ & $M S E(\widehat{\theta})$ & $M S E(\widehat{\lambda})$ \\
\hline & 15 & 0.0185 & 0.1548 & 0.0305 & 0.2507 \\
\hline & 25 & 0.0148 & 0.1498 & 0.0224 & 0.2276 \\
\hline & 35 & 0.0117 & 0.0129 & 0.0152 & 0.1252 \\
\hline$(0.15,0.35,0.65,0.3)$ & 45 & 0.0114 & 0.0155 & 0.0135 & 0.0865 \\
\hline & 55 & 0.0162 & 0.0379 & 0.0125 & 0.0643 \\
\hline & 65 & 0.0052 & 0.0208 & 0.0065 & 0.0316 \\
\hline & 75 & 0.0093 & 0.0137 & 0.0059 & 0.0138 \\
\hline & & & & & \\
\hline & 15 & 0.0340 & 0.0760 & 0.4058 & 0.0847 \\
\hline & 25 & 0.0216 & 0.0391 & 0.2918 & 0.0584 \\
\hline & 35 & 0.0212 & 0.0332 & 0.1807 & 0.0452 \\
\hline & 45 & 0.0176 & 0.0292 & 0.1662 & 0.0338 \\
\hline$(0.3,0.6,2,0.7)$ & 55 & 0.0098 & 0.0191 & 0.0899 & 0.0260 \\
\hline & 65 & 0.0017 & 0.0063 & 0.0337 & 0.0067 \\
\hline & 75 & 0.0012 & 0.0056 & 0.0246 & 0.0087 \\
\hline & & & & & \\
\hline & 15 & 0.1521 & 0.4083 & 0.5003 & 0.1452 \\
\hline & 25 & 0.0335 & 0.0693 & 0.4104 & 0.1335 \\
\hline & 35 & 0.0326 & 0.0497 & 0.3951 & 0.0841 \\
\hline & 45 & 0.0227 & 0.0393 & 0.3170 & 0.0618 \\
\hline & 55 & 0.0180 & 0.0600 & 0.2866 & 0.0417 \\
\hline & 65 & 0.0240 & 0.0546 & 0.2468 & 0.0390 \\
\hline & 75 & 0.0232 & 0.0485 & 0.1470 & 0.0163 \\
\hline & & & & & \\
\hline & & & & \\
\hline & & & & &
\end{tabular}

$\widehat{\beta}, \widehat{\theta}$, and $\widehat{\lambda}$. Using 33 , we approximate $100(1-\gamma)$ confidence intervals for $\alpha, \theta$, and $\lambda$ are determined respectively as

$$
\widehat{\alpha} \pm z_{\frac{\gamma}{2}} \sqrt{\widehat{V_{\alpha \alpha}}}, \widehat{\theta} \pm z_{\frac{\gamma}{2}} \sqrt{\widehat{V_{\theta \theta}}} \text {, and } \widehat{\lambda} \pm z_{\frac{\gamma}{2}} \sqrt{\widehat{V_{\lambda \lambda}}}
$$

where $z_{\gamma}$ is the upper $100 \gamma$ the percentile of the standard normal distribution. The following table represents the mean square error (MSEs) of the MLEs.

Table 1 The mean square errors of the MLEs.

We noticed from the above Table 1 that all MSEs decrease as the sample size increases, while they increase with increasing of the true parameter.

\section{APPLICATIONS}

In this section two real data sets are considered to see which one of distributions is more appropriate to the data set for some MLEs of parameters.Such as the transmuted generalized linear exponential distribution(TGLED), the Linear exponential distribution, Transmuted Linear exponential distribution, Transmuted Raylight distribution, Raylight distribution (LED, TLED, TRD, RD). To test the goodness-of-fit of selected distributions in each example, we calculated the Kol-mogorov Smirnov (K-S) distance test statistic and its correspondicorresponding $\mathrm{p}$-value.

EXAMPLE 1. Consider the data given by Abouammoh et al. (1994) which represent 40 patients suffering from leukemia from one of the Ministry of Health Hospitals in Saudi Arabia. The ordered lifetimes (in days) are given in Table 2.

Table 2 Lifetimes of 40 patients suffering from leukemia.

$\begin{array}{llllllll}115 & 181 & 255 & 418 & 441 & 461 & 516 & 739 \\ 743 & 789 & 807 & 865 & 924 & 983 & 1024 & 1062 \\ 1063 & 1165 & 1191 & 1222 & 1222 & 1251 & 1277 & 1290 \\ 1357 & 1369 & 1408 & 1455 & 1478 & 1549 & 1578 & 1578 \\ 1599 & 1603 & 1605 & 1696 & 1735 & 1799 & 1815 & 1852\end{array}$

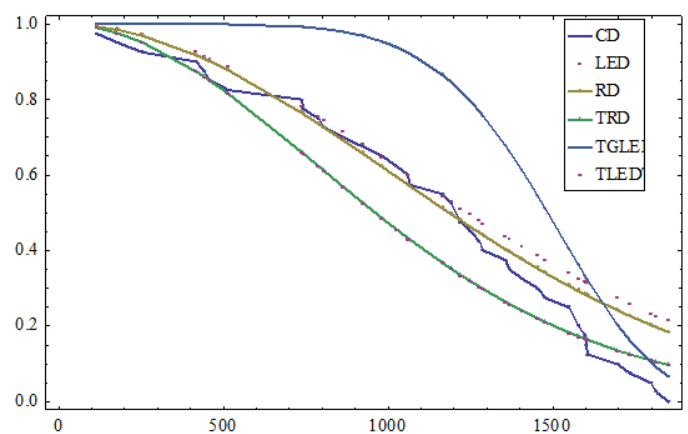

Table 3.The $K$ - $S$ distance test statistic and corresponding $p$-values.

$\begin{array}{ccc}\text { Modeling distribution } & K \text {-S test } & p \text {-value } \\ \text { TGLED } & 0.3554414 & 0.000049 \\ \text { LED } & 0.213624 & 0.044205 \\ \text { TLED } & 0.2094859 & 0.051105 \\ \text { TRD } & 0.207288 & 0.055130 \\ \text { RD } & 0.184278 & 0.116136\end{array}$

Fig. 4 is provided to compare the empirical reliability functions against the theoretical reliability functions of the modeling distributions.

Fig.4. Empirical and estimated survival functions of the LED, RD, TRD,TGLED and TLED models for (Leukemia data)

EXAMPLE 2. The lifetimes of 50 devices that were provided by (Aarset, 1987) are given in Table 4.

Table 4 Lifetimes of 50 devices.

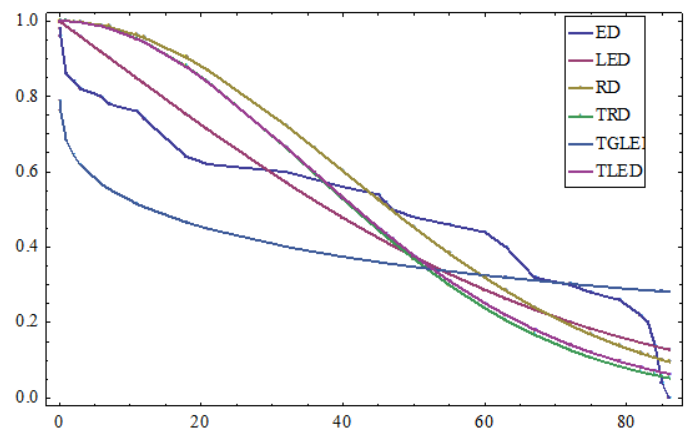

$\begin{array}{llllllll}0.1 & 0.2 & 1.0 & 1.0 & 1.0 & 1.0 & 1.0 & 2.0 \\ 3.0 & 6.0 & 7.0 & 11.0 & 12.0 & 18.0 & 18.0 & 18.0 \\ 18.0 & 18.0 & 21.0 & 32.0 & 36.0 & 40.0 & 45.0 & 46.0 \\ 47.0 & 50.0 & 55.0 & 60.0 & 63.0 & 63.0 & 67.0 & 67.0 \\ 67.0 & 67.0 & 72.0 & 75.0 & 79.0 & 82.0 & 82.0 & 83.0 \\ 84.0 & 84.0 & 84.0 & 85.0 & 85.0 & 85.0 & 85.0 & 85.0 \\ 86.0 & 86.0 & & & & & & \end{array}$

Table 5.The $K$ - $S$ distance test statistic and corresponding $p$-values.

Modeling distribution $\quad K$-S test $\quad p$-value

$\begin{array}{ccc}\text { TGLED } & 0.2812918 & 0.000542 \\ \text { LED } & 0.176099 & 0.0793432 \\ T L E D & 0.23746893 & 0.005777640 \\ T R D & 0.23832517 & 0.005878 \\ R D & 0.26237234 & 0.0015858\end{array}$

Fig. 5 is provided to compare the empirical reliability functions against the theoretical reliability functions of the modeling distributions. 
Fig. 5. Empirical and estimated survival functions of the $L E D, R D$, TRD, TGLED and TLED models for (Leukemia data) Lifetimes of 40 patients suffering from Leukemia.

By calculating $K$-S test and corresponding p-values for TGLED and some special cases as LED, RD, TRD and TLED for mentioned two survival data examples we can say that the distribution of TGLED can be appropriate to deal with Life data under different levels of significant. Table 3 and Table 5 contain some of the results that have been obtained for the two mentioned previous examples.

\section{REFERENCES}

1 Aarset, M.V.(1987). How to identify bathtub hazard rate. IEEE Transactions on Reliability R-36, 106108.

2 Abouammoh, A., Abdulghani, S., Qamber, I.(1994). On partial orderings and testing of new better than renewal used classes. Reliability Engineering andSystem Safety 43, 3741.

3 Aryal, G. R. and Tsokos, C. P.(2011). Transmuted Weibull distribution: A Generalization of the Weibull Probability Distribution. European Journal of Pure and Applied Mathematics, 4(2), 89102.

4 Aryal. G. R. and Tsokos, C. P.(2009). On the transmuted extreme value distribution with applications. Nonlinear Analysis: Theory, Methods and applications, Vol. 71, 1401-1407.

5 Aryal, G. R. (2013). Transmuted Log-Logistic Distribution. J. Stat. Appl. Pro. 2, No. 1, 11-20.

6 Elbatal, I. ( 2013). Transmuted modified inverseWeibull Distribution: AGeneralization of the Modified inverse Weibull Probability Distribution. International Journal of Mathematical Archive-4(8), 117-129.

7 Elbatal, I. and Aryal. G. R. .On the Transmuted AdditiveWeibull Distribution.Austrian Journal of Statistics (To Appear).

8 Lai, C.D., Xie, M., Murthy, D.N.P. (2001). Bathtub shaped failure rate distributions. In: Balakrishnan, N., Rao, C.R. (Eds.), Handbook in Reliability, vol. 20. 69104.

9 Mahmoud, M.A.Wand Alam,F. (2010). The generalized linear exponential distribution, Statist. Probabil. Lett. 80 ,1005-1014.

10 Merovci, F.and Elbatal, I.( 2013). Transmuted lindley-Geometric distribution and its applications.Stat.ME.arXiv:1309:3774V1.(To submitted ).

11 Muhammad, K.S. and Robert, K.( 2013). Transmuted Modified Weibull Distribution: A Generalization of the Modified Weibull Probability Distribution. European Journal of Pure and Applied Mathematics. 6(1), 66-88.

12 Shaw,W. and Buckley,I. (2007).The alchemy of probability distributions: beyond Gram- Charlier expansions and a skewkurtotic- normal distribution from a rank transmutation map.

13 Zhang,T , Xie, M,Tang,L and Ng,S (2005) Reliability and Modeling of Systems Integrated with Firmware and hardware, Int. J. Reliab. Quality Safety Eng. 12 (3), 227-239. 